\title{
Simulating Si multiple tunnel junctions from pinch-off to ohmic conductance
}

\author{
Heinz-Olaf Müller ${ }^{\mathrm{a}, *}$, David A. Williams ${ }^{\text {a }}$, Hiroshi Mizuta ${ }^{\mathrm{a}}$, Zahid A.K. Durrani ${ }^{\mathrm{b}}$ \\ ${ }^{a}$ Hitachi Cambridge Laboratory, Cavendish Laboratory, Madingley Road, Cambridge CB3 OHE, UK \\ ${ }^{\mathrm{b}}$ Microelectronics Research Centre, Cavendish Laboratory, Madingley Road, Cambridge CB3 OHE, UK
}

\begin{abstract}
Due to their compatibility with CMOS, multiple tunnel junctions (MTJs) are giving rise to an increasing interest in Coulomb blockade silicon devices, along with a higher demand for simulation. Whereas the operating principle has been known for a number of years, here we present new simulation results on MTJs, including geometric island size and island separation. Application of MTJ in a memory cell is discussed. (C) 2000 Elsevier Science S.A. All rights reserved.
\end{abstract}

Keywords: Simulation; Si multiple tunnel junctions; Pinch-off; Ohmic conductance

\section{Basics}

The concept of multiple tunnel junctions (MTJs) has been used since 1993. From the beginning [1] there were high expectations, especially based on the advantage of MTJs to combine Coulomb blockade [2] and CMOS (complementary metal-oxide semiconductor). It is not only that CMOS is the mainstream technology in today's electronics, but also that CMOS and SET (single electron tunneling) electronics complement each other very favorably (Table 1). The steadily growing interest in MTJ systems is also indicated by the increasing number of papers on MTJs per year (Fig. 1).

Physically, an MTJ is a narrow (approximately 50 $\mathrm{nm}$ ), highly doped (approximately $1 \times 10^{19} \mathrm{~cm}^{-3}$ ) silicon wire with an attached gate. This means that usually e-beam lithography is required to achieve the small dimension, sometimes followed by an oxidation step for the same reason. The operating principle was already qualitatively understood along with the first experiments [1]: the dopants cause potential fluctuations which affect the conduction band electrons (Fig. 2). By means of the gate, the Fermi energy can be adjusted to a regime where electronic transport is by single electron tunneling. The narrow width of the MTJ restricts the number of the tunneling channels, resulting in quasi-

\footnotetext{
* Corresponding author.

E-mail address: hom@phy.cam.ac.uk (H.-O. Müller)
}

one-dimensional transport. Fig. 2 shows that for very negative gate voltage the MTJ is 'pinched off', i.e. no electron transport occurs at all, and for large gate voltage the MTJ becomes conductive ('ohmic').

A typical set of characteristics is shown in Fig. 3. Pinch-off is observed for low gate voltage, before the Coulomb blockade oscillations set in. These oscillations are rather irregular (compared with results of metallic systems [3]) and their oscillation period decreases with increasing gate voltage.

\section{Simulation}

In terms of the 'orthodox theory' of single electron tunneling [4] an MTJ can be understood as an one-dimensional array of quantum dots with changing number and sizes of the dots as the applied gate voltage is altered. Hence, pinch-off translates into many small dots with prohibitive dot separation and ohmic conductance occurs after the last island disappears in the Fermi sea.

The simplest case is that of a single dot (double junction, Fig. 4). This situation occurs close to the ohmic regime. The novel feature of our 'blocking dot' model is the size variation of this dot caused by a varying gate voltage. For the quantitative description of this effect we use an experiment with an MTJ which allows the determination of the Coulomb blockade 
Table 1

Advantages and disadvantages of SET electronics vs. CMOS electronics

\begin{tabular}{ll}
\hline SET & CMOS \\
\hline Low power & High power \\
High density & Low density \\
High impedance & Low impedance \\
Poor gain & High gain \\
Low drivability & High drivability \\
\hline
\end{tabular}

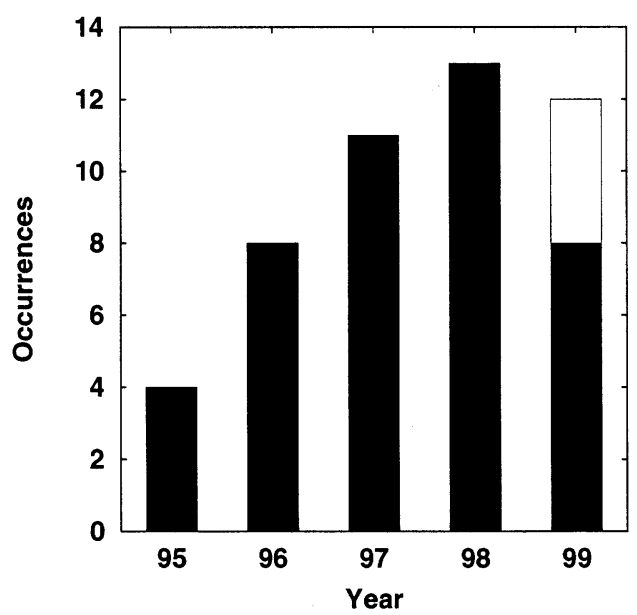

Fig. 1. The number of papers on experiments with MTJs, according to BIDS (http://www.bids.ac.uk). A steady increase is observed since the introduction of the term (as of September 1999).

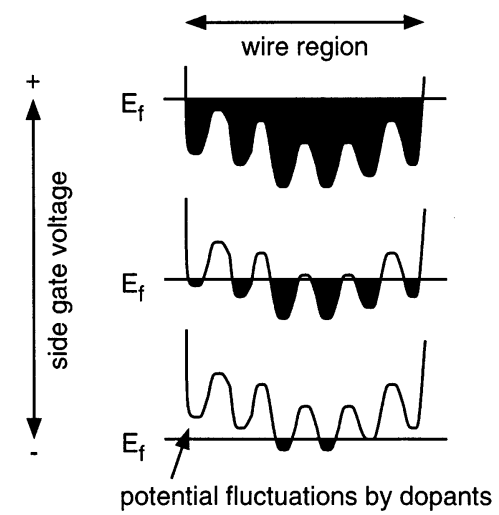

Fig. 2. Operating principle of an MTJ. Doping causes electronic potential fluctuations. Since the Fermi energy can be shifted by the gate. a working point can be reached, where transport is governed by single electron tunneling from dot to dot.

voltage $V_{\mathrm{b}}$ and, via $C_{\Sigma}=e / V_{\mathrm{b}}$, the determination of the overall capacitance of the dot. On the other hand we can calculate this capacitance via an analytical formula from the dot radius $r$, the center-to-center dot separation $c$, and the dot-gate separation $d[5]$ :

$C_{\Sigma}=2 C_{\mathrm{dd}}+2 C_{\mathrm{dg}}+C_{\mathrm{sc}}$
$C_{\mathrm{dd}}=4 \pi \varepsilon r \sinh \beta \sum_{n=1}^{\infty} \operatorname{cosech} 2 n \beta$

dot - dot capacitance

$C_{\mathrm{dg}}=4 \pi \varepsilon r \sinh \alpha \sum_{n=1}^{\infty} \operatorname{cosech} n \alpha$

dot - gate capacitance

$C_{\mathrm{sc}}=4 \pi \varepsilon r \sinh \beta \sum_{n=1}^{\infty} \operatorname{cosech}(2 n-1) \beta$

dot stray capacitance

using $\cosh \alpha=d / r$ and $\cosh \beta=c /(2 r)$. Consistent with the model outlined above we assume that $c$ and $d$ are independent of $V_{\mathrm{sg}}$, whereas $r$ is a function of the gate voltage. Given a functional dependence of $r\left(V_{\mathrm{sg}}\right)$ which we can fit to the experimental data, we can determine not only the parameters of this function, but also $c$ and $d$. Due to the considerable gate oxide thickness, $d$ is in general larger than $c$ or $r$. Therefore, we fix this parameter to a reasonable value and determine only $c$ and $r\left(V_{\mathrm{sg}}\right)$ from the fit.

As is seen in Fig. 5, the fit reproduces the capacitance values of $C_{\Sigma}$ for three very different test functions $r\left(V_{\mathrm{sg}}\right)$ quite well and cannot discriminate between the approaches. Therefore, two additional checks are used. Firstly, the dot size $r$ should be within a reasonable

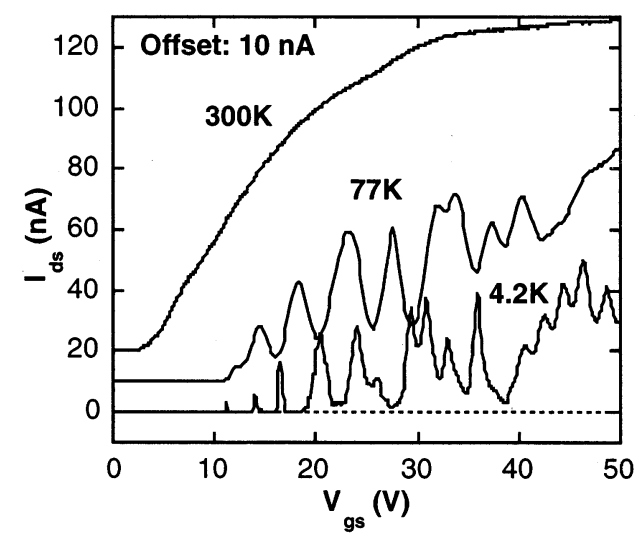

Fig. 3. Temperature dependence of the current through an MTJ. Source-drain voltage is $20 \mathrm{mV}$ in this case and the MTJ parameters are doping $1 \times 10^{19} \mathrm{~cm}^{-3}$, width $40 \mathrm{~nm}$, height $50 \mathrm{~nm}$, with subsequent oxidation to reduce the effective wire cross section by about 10 $\mathrm{nm}$.

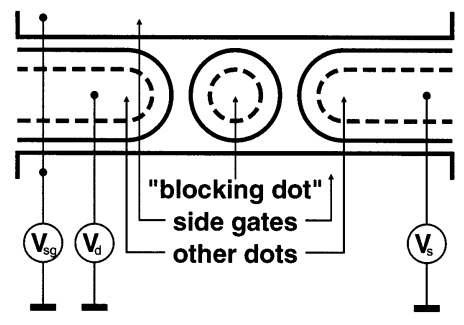

Fig. 4. The blocking dot model. A single dot is connected to continuous leads (double junction). The size of the dot varies with the applied side gate voltage. 


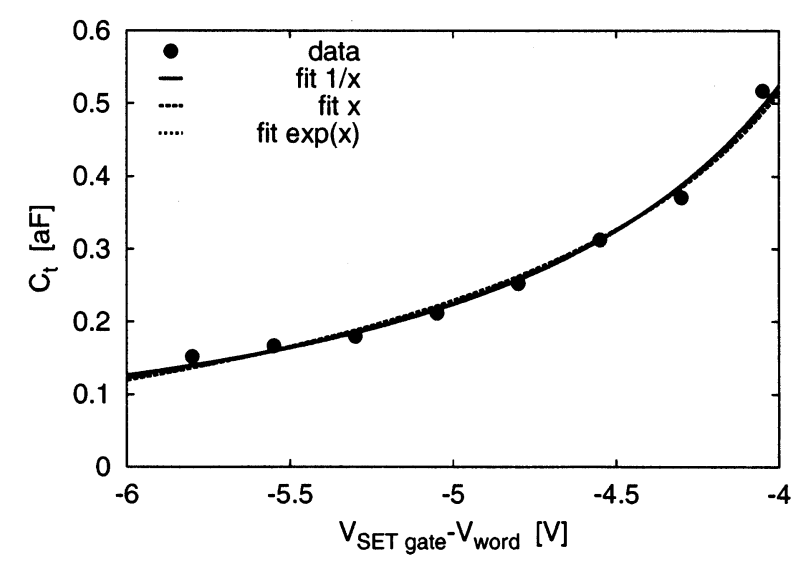

Fig. 5. The fit of the calculated capacitance values to experimental data. Three different functional dependencies $r\left(V_{\mathrm{sg}}\right)$ result in similar behavior of the overall capacitance $C_{\Sigma}$.

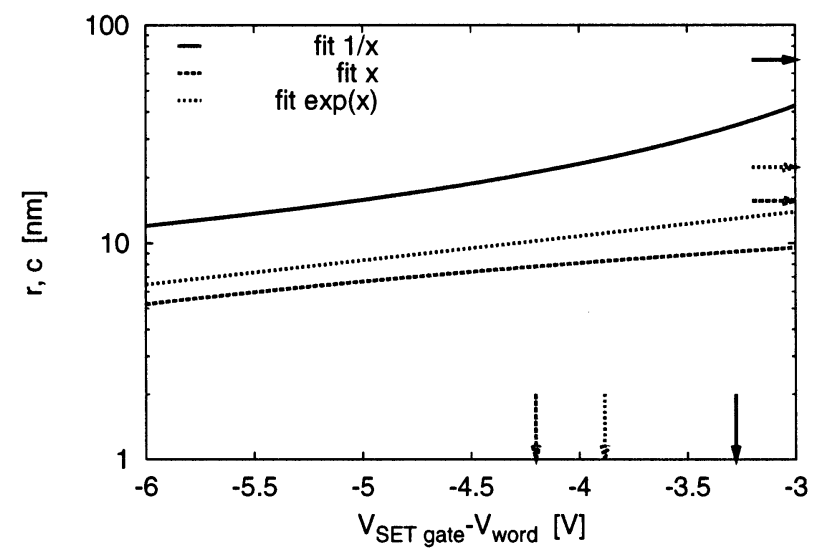

Fig. 6. Plot of the fit results in terms of $r$ and $c$. the arrows indicate $V_{1 / 2}$, at which the dot and the leads overlap (horizontal axis) and $c$ (vertical axis).

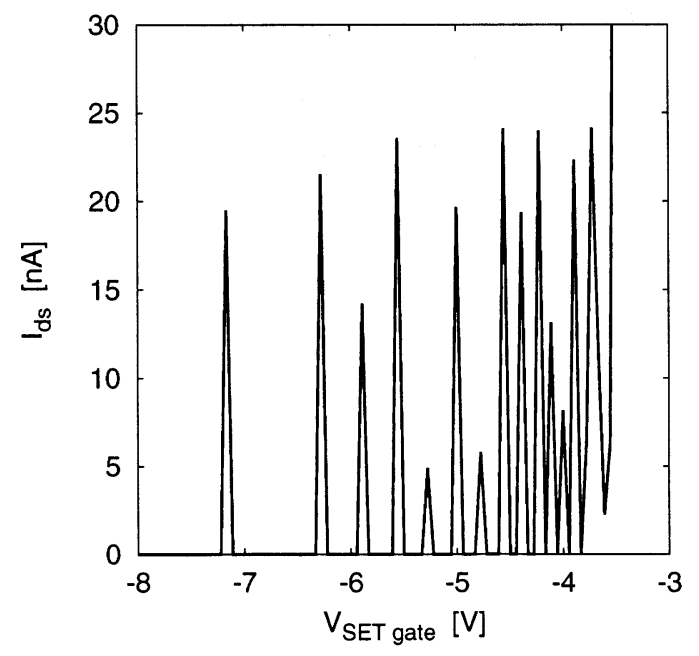

Fig. 7. Simulation of Coulomb blockade oscillations in a MTJ using the blocking dot model as outlined in this paper. Note the reduction of the oscillation period with increasing gate voltage.

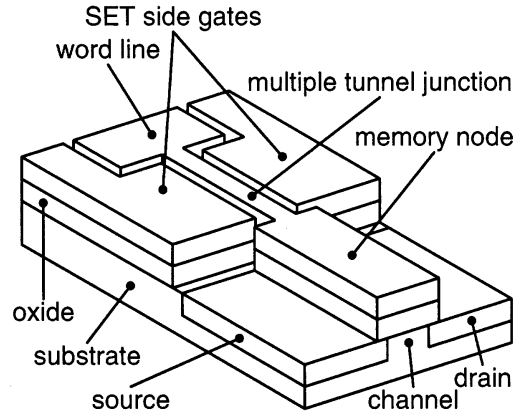

Fig. 8. Lateral single electron memory cell. The memory node is coupled to the word line via an MTJ.

range, for instance not larger than the geometric size of the MTJ as determined from scanning electron microscopy (SEM) pictures. Secondly, the voltage $V_{1 / 2}$ at which the last dot dissolves can be determined from $2 r\left(V_{1 / 2}\right)=c$ and compared to the experimental crossover to ohmic behavior. This is illustrated in Fig. 6. It appears that the exponential dependence $r=c /$ $2 \exp \left(A\left(V_{\mathrm{sg}}-V_{1 / 2}\right)\right)$ gives the best results using $c \approx 22$ nm and $A \approx 0.256 / V$. A cross check with another (but corresponding) experiment yielded similar results. Note in addition that the exponential dependence is expected for the screened Coulomb potential of a dopant atom.

We also simulated the effect of a second dot in series with the first one discussed above resulting in an increased blockade. To this end we employ the same exponential dependence as used above and the same value of $A$, however a lower value of $V_{1 / 2}$, labeled $V_{2 / 3}$. Hence we find ohmic operation for $V_{\mathrm{sg}}>V_{1 / 2}$, single dot oscillations for $V_{1 / 2}>V_{\mathrm{sg}}>V_{2 / 3}$ and initial pinch off for $V_{\mathrm{sg}}<V_{2 / 3}$. More and more dots are expected to occur in the current path for decreasing gate voltage thus increasingly obstructing electronic transport.

\section{Results}

Using the model of the last section we were able to compute the Coulomb blockade oscillations of Fig. 7. For very negative gate voltage the MTJ is pinched off and for high values of the gate voltage the conductance jumps up to its ohmic value. In between these two extreme cases oscillations are observed. The period decreases with increasing gate voltage; very much corresponding to the experimental data of Fig. 3. Within our model this change is easily explained by the increasing dot size and thus dot capacitance.

We have also checked for additional effects like the influence of the dot's stray capacitance, which turns the $I-V$ characteristic into an asymmetric one (for nonsymmetric bias), similar to the behavior of the ultrasmall double junction [6]. 
One application of MTJs envisioned from the very beginning is their use in memory cells which was achieved only recently [7] (see Fig. 8). In the lateral single electron memory cell used the MTJ connects the write word line to the memory node. The read out uses a MOSFET transistor underneath the memory node thus obtaining gain cell operation (this setup complicates the simulation to some extend as it requires a simulator that is capable of dealing with both the single electron part and the MOSFET part of the cell $[8,9])$. In difference to the experiment considered above, the potential of the memory node is usually not at ground level. A change in the memory node potential thus unavoidably influences the potential difference between the MTJ and the external gate bias. In turn, the size of the $\operatorname{dot}(\mathrm{s})$ forming the MTJ change as well. This feed back situation causes fingerprints of Coulomb oscillations to occur in the current through the MTJ at a fixed gate voltage. In the hysteresis characteristics of the memory cell these fingerprints are small current steps. These steps again follow the pattern discussed above for the Coulomb oscillations: they become fewer (and larger) for more negative gate voltage.

However, quantitative simulation of MTJ is still not achieved. The reason for this is the lack of a model for the tunneling resistance. Similar to the capacitance discussed above, the resistance would depend on the size $r$ of the dots. But it would also depend on the shape and height of the potential between the dots, which is generally hard to access.

\section{Conclusions}

We present a blocking dot model for MTJs and show how to determine it's parameters by a fit procedure.
Having set up the model, we are able to simulate MTJs on a new level. For instance, the experimentally observed change of the period of Coulomb blockade oscillations in MTJs is easily explained. The model allows for a geometric insight into the MTJ's geometry as well.

We also discuss how to use the model in connection with a single electron memory cell, the operation of which was recently demonstrated. The shortcomings of our model are discussed as far as quantitative analysis is concerned.

\section{Acknowledgements}

This work was performed within the ESPRIT MELAPI project FASEM (Fabrication and Architecture of Single-Electron Memories).

\section{References}

[1] K. Nakazato, H. Ahmed, Adv. Mater. 5 (1993) 668.

[2] M.H. Devoret, H. Grabert, NATO ASI Ser. B Phy. 294 (1992) $1-19$.

[3] D. Esteve, NATO ASI Ser. B Phy. 294 (1992) 109-138.

[4] D.V. Averin, K.K. Likharev, in: B.L. Altshuler, P.A. Lee, R.A. Webb (Eds.), Mesoscopic Phenomena in Solids, Modern Problems in Condensed Matter Sciences, vol. 30, Elsevier, Amsterdam, 1991, pp. 173-271.

[5] W.R. Smythe, Static and Dynamic Electricity, McGraw-Hill, New York, 1968.

[6] G.-L. Ingold, P. Wyrowski, H. Grabert, Z. Phys. B85 (1991) 443.

[7] Z.A.K. Durrani, A.C. Irvine, H. Ahmed, K. Nakazato, Appl. Phys. Lett. 74 (1999) 1293.

[8] S. Amakawa, et al., IEICE Trans. Electron. 81C (1998) 21.

[9] M. Kirihara, K. Nakazato, N.I. Wagner, Jpn. J. Appl. Phys. 38/1 (1999) 2028. 